\title{
Nicotinamide phosphoribosyltransferase-related signaling pathway in early Alzheimer's disease mouse models
}

\author{
SANLI XING* , YIRAN HU* ${ }^{*}$ XUJIAO HUANG, DINGZHU SHEN and CHUAN CHEN \\ Shanghai Geriatric Institute of Chinese Medicine, Shanghai University of Traditional Chinese Medicine, \\ Shanghai 200031, P.R. China
}

Received April 8, 2019; Accepted October 2, 2019

DOI: $10.3892 / \mathrm{mmr} .2019 .10782$

\begin{abstract}
Alzheimer's disease (AD) is a neurodegenerative disease of the central nervous system that is characterized by progressive cognitive dysfunction and which ultimately leads to dementia. Studies have shown that energy dysmetabolism contributes significantly to the pathogenesis of a variety of aging-associated diseases and degenerative diseases of the nervous system, including AD. One focus of research thus has been how to regulate the expression of nicotinamide phosphoribosyltransferase (NAMPT) to prevent against neurodegenerative diseases. Therefore, the present study used 6-month-old APPswe/PS1 $\triangle \mathrm{E} 9$ (APP/PS1) transgenic mice as early AD mouse models and sought to evaluate nicotinamide adenine dinucleotide $\left(\mathrm{NAD}^{+}\right)$and FK866 (a NAMPT inhibitor) treatment in APP/PS1 mice to study NAMPT dysmetabolism in the process of $\mathrm{AD}$ and elucidate the underlying mechanisms. As a result of this treatment, the expression of NAMPT decreased, the synthesis of ATP and NAD ${ }^{+}$became insufficient and the $\mathrm{NAD}^{+} / \mathrm{NADH}$ ratio was reduced. The administration of $\mathrm{NAD}^{+}$alleviated the spatial learning and memory of APP/PS1 mice and reduced senile plaques. Administration of $\mathrm{NAD}^{+}$ may also increase the expression of the key protein NAMPT and its related protein sirtuin 1 as well as the synthesis of $\mathrm{NAD}^{+}$. Therefore, increasing NAMPT expression levels may promote $\mathrm{NAD}^{+}$production. Their regulation could form the basis for a new therapeutic strategy.
\end{abstract}

Correspondence to: Mr. Chuan Chen, Shanghai Geriatric Institute of Chinese Medicine, Shanghai University of Traditional Chinese Medicine, 365C South Xiangyang Road, Shanghai 200031, P.R. China

E-mail: article_chenchuan@163.com

${ }^{*}$ Contributed equally

Key words: Alzheimer's disease, nicotinamide adenine dinucleotide, nicotinamide phosphoribosyltransferase

\section{Introduction}

Alzheimer's disease (AD) is a neurodegenerative disease of the central nervous system that is characterized by progressive cognitive dysfunction, ultimately leading to dementia. While the pathological hallmarks are mainly the formation of extracellular senile plaques, intracellular neurofibrillary tangles and neuronal loss, its pathogenesis has not been fully elucidated (1). Previous studies have demonstrated that energy dysmetabolism contributes significantly to the pathogenesis of a variety of aging-associated diseases and degenerative disease of the nervous system, including AD $(2,3)$. With aging, the imbalance of energy metabolism in the brain is intensified, which promotes amyloid (A) $\beta$ production. $A \beta$ further induces energy metabolism disorder in the brain $(4,5)$. When the energy metabolism of neurons in the brain is deregulated, ATP synthesis is insufficient and leads to irreversible damage to neuronal structure and function $(6,7)$. Since research has demonstrated that energy disorders of the brain have an important impact on the development of $\mathrm{AD}$, improving the energy metabolism of nerve cells in the brain is expected to delay the pathogenesis of AD (8-10).

Nicotinamide adenine dinucleotide $\left(\mathrm{NAD}^{+}\right)$is an important coenzyme in the redox reaction, which can participate in energy synthesis through glycolysis, the tricarboxylic acid cycle and mitochondrial oxidative phosphorylation (10). The majority of $\mathrm{NAD}^{+}$is synthesized by a salvage pathway from the conversion of nicotinamide to a nicotinamide mononucleotide, which is subsequently converted to $\mathrm{NAD}^{+}(11)$. Nicotinamide phosphoribosyltransferase (NAMPT) is the rate-limiting enzyme in the $\mathrm{NAD}^{+}$rescue pathway and is involved in energy metabolism by regulating $\mathrm{NAD}^{+}$synthesis (12), maintaining the homeostasis of energy metabolism in the brain and thereby increasing the expression of $\mathrm{NAD}^{+}$(the level of NAMPT has been proven to regulate positively $\mathrm{NAD}^{+}$levels) (13). However, few studies to date have directly explored the potential therapeutic effect of targeting NAMPT and $\mathrm{NAD}^{+}$biosynthesis, despite it being known that $\mathrm{NAD}^{+}$is the starting point of the majority of key metabolic pathways and therefore the key governor of cellular aging and age-related processes $(14,15)$. How to regulate the expression of NAMPT to protect against neurodegenerative diseases such as AD has become the focus of recent research. 
The present study used 6-month-old APPswe/PS1 $\Delta \mathrm{E} 9$ (APP/PS1) transgenic mice as early AD mouse models and observed the mechanism of NAMPT-related pathways in the process of $\mathrm{AD}$.

\section{Materials and methods}

Ethics statement. All procedures were performed in accordance with the Guide for and Use of Medical Laboratory Animals (Ministry of Health China, 1998) (16) and were approved by the Shanghai University of Traditional Chinese Medicine (Shanghai, China) Laboratory Animal Care and Use Committee (no. PZSHUTCM19011807).

APP/PS1 transgenic mice and drug treatment. In total, 18 male APP/PS1 transgenic mice (age: 24 weeks; weight: $26 \pm 3 \mathrm{~g}$ ) and 6 male C57BL/6 mice (age: 24 weeks; weight: $27 \pm 2 \mathrm{~g}$ ) were used in the present study. The APP/PS1 mouse strain is a double-transgenic hemizygote that expresses a chimeric mouse/human amyloid precursor protein and mutant human presenilin-1. These transgenic mice were used as they develop behavioral and pathology features of $\mathrm{AD}$ similar to patients and have been used in previous $\mathrm{AD}$ studies worldwide (17-19). Separately, C57BL/6 mice were used as age-matched controls. The APP/PS1 transgenic mice were purchased from the Model Animal Research Center of Nanjing University, while C57BL/6 mice were obtained from the Experimental Animal Center of Shanghai Academy. The $\mathrm{NAD}^{+}$group were intraperitoneally injected with $\mathrm{NAD}^{+}$ (30 mg/kg, Sigma-Aldrich; Merck KGaA) at 20 weeks of age and once every other day for 4 weeks. The FK866 (NAMPT inhibitor) group were intraperitoneally injected with FK866 $(1 \mathrm{mg} / \mathrm{kg}$, Sigma-Aldrich; Merck KGaA) at 20 weeks of age and once every other day for 4 weeks. The mice were housed in a controlled specific-pathogen-free environment $\left(22 \pm 3^{\circ} \mathrm{C}\right.$; $60 \%$ relative humidity; 12 -h light/dark cycle) with ad libitum access to water and food.

Morris water maze test. Spatial learning and memory of the mice were assessed using a Morris water maze test (MWM) according to a previous study (20) with minor modifications. The water was made opaque with titanium dioxide and water temperature was kept at $22 \pm 2^{\circ} \mathrm{C}$. A platform was placed $1 \mathrm{~cm}$ under the surface of the water. In the hidden platform experiment, mice received 4 training trials per day for 5 consecutive days. On the first trial of the first day, mice were placed on the platform for $10 \mathrm{sec}$, after which they were placed in the water. The pool area was conceptually divided into four quadrants of equal size. Taking the quadrant of the platform as the first quadrant, the second and fourth quadrants were taken as the starting point for 2 trials and the mice were placed facing the pool wall. If a mouse failed to find the platform in $70 \mathrm{sec}$, it was gently guided to the platform location and allowed to stay on it for $30 \mathrm{sec}$. The time to find the platform was recorded as the escape latency. The experiments were recorded with a camera connected a video recorder and a computerized tracking system, with the automatic timer set to $70 \mathrm{sec}$.

In the probe trials, at $24 \mathrm{~h}$ after the last training trial, the platform was removed and the mice were placed at the second and fourth quadrants, and the time of the target quadrant (first quadrant) and the number of times crossing the location that previously contained the platform within $70 \mathrm{sec}$ were recorded.

Thioflavin S staining. Mice were anesthetized with 5\% chloral hydrate at a dose of $400 \mathrm{mg} / \mathrm{kg}$ intraperitoneally and saline solution was used for perfusion through the heart, which was followed by $4 \%$ paraformaldehyde. The brains of the mice were then removed, fixed in $4 \%$ paraformaldehyde for $24 \mathrm{~h}$ at room temperature and immersed in $30 \%$ sucrose until they sank. The brain tissue was serially sectioned at a $30-\mu \mathrm{m}$ thickness using a HM1950 freezing microtome. The sections were permeabilized in xylene for 10 min, dehydrated using anhydrous ethanol for $5 \mathrm{~min}$, and then stained with $1 \%$ thioflavin $\mathrm{S}$ (Sigma-Aldrich; Merck KGaA) at $37^{\circ} \mathrm{C}$ for $30 \mathrm{~min}$. The stained samples were differentiated in $70 \%$ alcohol solution for $5 \mathrm{~min}$, mounted with glycerol gel, and observed using fluorescence microscopy (magnification, 200x; Olympus BX51; Olympus Corporation).

$N A D^{+} / \mathrm{NADH}$ analysis. A NAD $/ \mathrm{NADH}$ quantification kit was used to determine $\mathrm{NAD}^{+} / \mathrm{NADH}$ levels (cat. no. k337-100; BioVision, Inc.), according to the manufacturer's instructions. Hippocampus tissue $(20 \mathrm{mg})$ was washed with precooled phosphate-buffered saline (PBS), homogenized in $400 \mu \mathrm{l}$ of the $\mathrm{NAD}^{+} / \mathrm{NADH}$ extraction buffer and then centrifuged for $5 \mathrm{~h}$ at $18,000 \mathrm{xg}$ at $4^{\circ} \mathrm{C}$. The resulting supernatant was labeled as total $\mathrm{NAD}^{+}$sample $\left(\mathrm{NAD}^{+} \mathrm{t}\right)$. Subsequently, $200 \mu \mathrm{l}$ of the $\mathrm{NAD}^{+} \mathrm{t}$ sample was heated at $60^{\circ} \mathrm{C}$ for $30 \mathrm{~min}$ (to consume all $\mathrm{NAD}^{+}$from the sample, leaving only NADH to be analyzed). Following cooling on ice, the sample was centrifuged at $12,000 \mathrm{x} \mathrm{g}$ for $30 \mathrm{sec}$ at $4^{\circ} \mathrm{C}$ and the resulting supernatant was labeled as the NADH sample. $\mathrm{NAD}^{+} / \mathrm{NADH}$ extraction buffer was added to bring the volume to $50 \mu$ l. Subsequently, $100 \mu 1$ of enzyme reaction mix and $10 \mu l$ of NADH developer were added to each well, the mixture was incubated at room temperature for $2 \mathrm{~h}$ and the absorbance was measured using a microplate reader (wavelength: $450 \mathrm{~nm}$ ).

ATP content detection. Cortical or hippocampus tissue $(10 \mathrm{mg})$ was washed with precooled PBS, homogenized in $100 \mu \mathrm{l}$ of lysis buffer (cat. no. P0013B: Beyotime Institute of Biotechnology) and centrifuged at $12,000 \mathrm{xg}$ at $4^{\circ} \mathrm{C}$ for $5 \mathrm{~min}$. The resulting supernatant was transferred to a new Eppendorf tube and then the reaction solution (ATP Assay Buffer, ATP probe, ATP Converter and Develop Mix; Sigma-Aldrich; Merck KGaA) was added to bring the volume to $50 \mu$ l. The reaction solution was shaken in a shaker for $30 \mathrm{~min}$ at room temperature. To correct for the background of the samples, a well per sample was used as the sample background well. The absorbance was measured at a wavelength of $570 \mathrm{~nm}$ using a microplate reader.

Immunohistochemical staining. Following a behavioral test, three mice were randomly selected in each group. Following anesthesia, the brains were perfused with $4 \%$ paraformaldehyde for $4 \mathrm{~h}$ and then transferred to a $30 \%$ sucrose gradient at room temperature. After the brains had sunk to the bottom, continuous coronal sectioning was performed ( $30 \mu \mathrm{m}$ thickness) using a HM1950 freezing microtome. The sections were permeabilized 
in xylene for $10 \mathrm{~min}$ and dehydrated using anhydrous ethanol for $5 \mathrm{~min}$. The sections were washed with $0.01 \mathrm{M}$ of PBS and blocked with 5\% BSA (Sigma-Aldrich; Merck KGaA) for $1 \mathrm{~h}$ at room temperature. Then the sections were incubated with polyclonal anti-NAMPT antibody (1:200; cat. no. ab45890; Abcam) overnight at $4^{\circ} \mathrm{C}$. Following rinsing with PBS, sections were incubated with horseradish peroxidase (HRP)-conjugated anti-rabbit IgG antibody (1:500; cat. no. SA00001-2; ProteinTech Group, Inc.) for $1 \mathrm{~h}$ at $37^{\circ} \mathrm{C}$, washed with PBS, and streptavidin-biotin complex (Beyotime Institute of Biotechnology) was added. Then the sections were developed with 3,3'-diaminobenzidine for $10 \mathrm{~min}$ at room temperature and dehydrated with gradient alcohol, cleared with xylene, sealed with neutral gum and observed with using a light microscope (magnification, x200; Olympus BX51; Olympus Corporation).

Western blot analysis. The isolated hippocampus tissue was collected, homogenized in lysis buffer (cat. no. P0013B; Beyotime Institute of Biotechnology), lysed on ice for $30 \mathrm{~min}$ and centrifuged at $12,000 \mathrm{x} \mathrm{g}$ for $20 \mathrm{~min}$ at $4^{\circ} \mathrm{C}$. Total protein concentration was determined using a Micro BCA Protein Assay kit (Thermo Fisher Scientific, Inc.). Then, $30 \mu \mathrm{g}$ of protein sample was heated at $95^{\circ} \mathrm{C}$ for 5 min and separated by $10 \%$ SDS-polyacrylamide gel electrophoresis, after which it was transferred onto a PVDF membrane. The membrane was blocked with $3 \% \mathrm{BSA}$ at $37^{\circ} \mathrm{C}$ for $1 \mathrm{~h}$. Following blocking, the membranes were incubated with primary antibodies (anti-NAMPT 1:500, cat. no. ab45890, Abcam; anti-SIRT1, 1:1,000, cat. no. ab110304, Abcam; and GAPDH, 1:1,000; cat. no. 5174; Cell Signaling Technology, Inc.) overnight at $4^{\circ} \mathrm{C}$. The membrane was washed with TBST three times for $10 \mathrm{~min}$ and then treated with HRP-labeled goat anti-rabbit secondary antibody (1:2,000; cat. no. SA00001-2; ProteinTech Group, Inc.) and HRP-labeled goat anti-mouse secondary antibody $(1: 2,000$; cat. no. SA00001-1; ProteinTech Group, Inc.) for $1 \mathrm{~h}$ at $37^{\circ} \mathrm{C}$. Blots were washed three times for $10 \mathrm{~min}$ in TBST. After incubation with enhanced chemiluminescence chromogenic solution (cat. no. P0018s; Beyotime Institute of Biotechnology) for $1 \mathrm{~min}$ at room temperature, quantification of the proteins of interest was determined relative to GAPDH using ImageJ software (version 1.4; National Institutes of Health).

Statistical analysis. Statistical analysis was performed using GraphPad software 6.0 (GraphPad Software, Inc.). Measurement data are presented as the mean \pm standard error of the mean. Two-way ANOVA followed by Bonferroni post hoc test was used to analyze group differences in the escape latency. A one-way ANOVA followed by LSD multiple-range test was used in the probe trial. For the non-normally distributed data or for data with heterogeneous variance, a Kruskal-Wallis test was used. $\mathrm{P}<0.05$ was considered to indicate a statistically significant difference.

\section{Results}

APP/PS1 mice exhibit impaired performance and effects of $N_{A D}^{+}$and FK866 treatment in the MWM tasks. Memory impairment in APP/PS1 transgenic mice was determined using the MWM test in terms of the escape latency. The results demonstrated that, with the increase in the number of training days, the escape latency gradually shortened. On the fifth day, the escape latency of the APP/PS1 group (AD) mice was significantly longer compared with the blank control group $(\mathrm{P}<0.01)$, suggesting that the learning and memory abilities of AD mice were significantly impaired. Furthermore, the escape latency of $\mathrm{NAD}^{+}$mice was significantly shorter than that of APP/PS1 mice after intraperitoneal injection of $\mathrm{NAD}^{+}$ $(\mathrm{P}<0.01)$; while, after FK866 intervention, there was no significant change in escape latency in the FK866 mice compared with the APP/PS1 mice (Fig. 1A).

In the space exploration experiment on the sixth day, after removing the platform it was found that APP/PS1 mice significantly reduced the target quadrant residence time $(\mathrm{P}<0.01)$ compared with control group mice, indicating that the spatial memory ability of AD mice was impaired. Following NAD ${ }^{+}$ treatment, the $\mathrm{NAD}^{+}$mice spent more time in the target quadrant compared with the APP/PS1 mice $(\mathrm{P}<0.01)$; however, there was no significant change in the target quadrant residence time of the mice in the APP/PS1 group compared with the FK866 group (Fig. 1B). In comparison with the control group, the number of platform crossings was significantly reduced in the APP/PS1 group $(\mathrm{P}<0.05)$ and the $\mathrm{NAD}^{+}$mice exhibited an increased number of platform crossings compared with the APP/PS1 mice, but these findings were not statistically significant $(\mathrm{P}>0.05)$. Overall, there was no significant change between the APP/PS1 group and FK866 group (Fig. 1C).

$\mathrm{NAD}^{+}$treatment reduces amyloid plaques of cortex and hippocampus in APP/PS1 transgenic mice. Staining with Thioflavin $\mathrm{S}$ for senile plaques in the cortex and hippocampus was conducted. As expected, the AD mice presented numerous plaques at 6 months of age as compared with the control mice in cortex and hippocampus; however, following $\mathrm{NAD}^{+}$treatment, scattered green fluorescent plaques were observed in the cortex and hippocampus of every group and, after FK866 injection, multiple green fluorescent plaques were also seen in the cortex and hippocampus in AD mice (Fig. 2).

$N^{+} D^{+}$treatment improves ATP content of cortex and hippocampus in APP/PS1 transgenic mice. ATP content in the cortex and hippocampus was measured in each group of mice. The results demonstrated that, compared with the control group, the ATP content in the cortex in the APP/PS1 group was significantly decreased $(\mathrm{P}<0.05)$, while, in comparison with the APP/PS1 model group, the ATP content in the cortex in the $\mathrm{NAD}^{+}$group was significantly increased $(\mathrm{P}<0.05)$. Following FK866 intervention, the ATP content of the cortex decreased compared with APP/PS1 mice, but without statistical significance $(\mathrm{P}>0.05)$. Additionally, in comparison with the control group, the ATP content in the hippocampus in the APP/PS1 group was significantly decreased $(\mathrm{P}<0.05)$, while, compared with the APP/PS1 model group, the ATP content in the hippocampus in the $\mathrm{NAD}^{+}$group was significantly increased $(\mathrm{P}<0.05)$. Following FK866 intervention, there was no significant change in the $\mathrm{NAD}^{+}$group compared with APP/PS1 model group (Fig. 3).

$N A D^{+}$treatment increases $N A D^{+}$level and $N A D^{+} / N A D H$ ratio in APP/PS1 transgenic mice. NAD ${ }^{+}$and NADH are essential coenzymes for cell energy metabolism. Under normal 
A
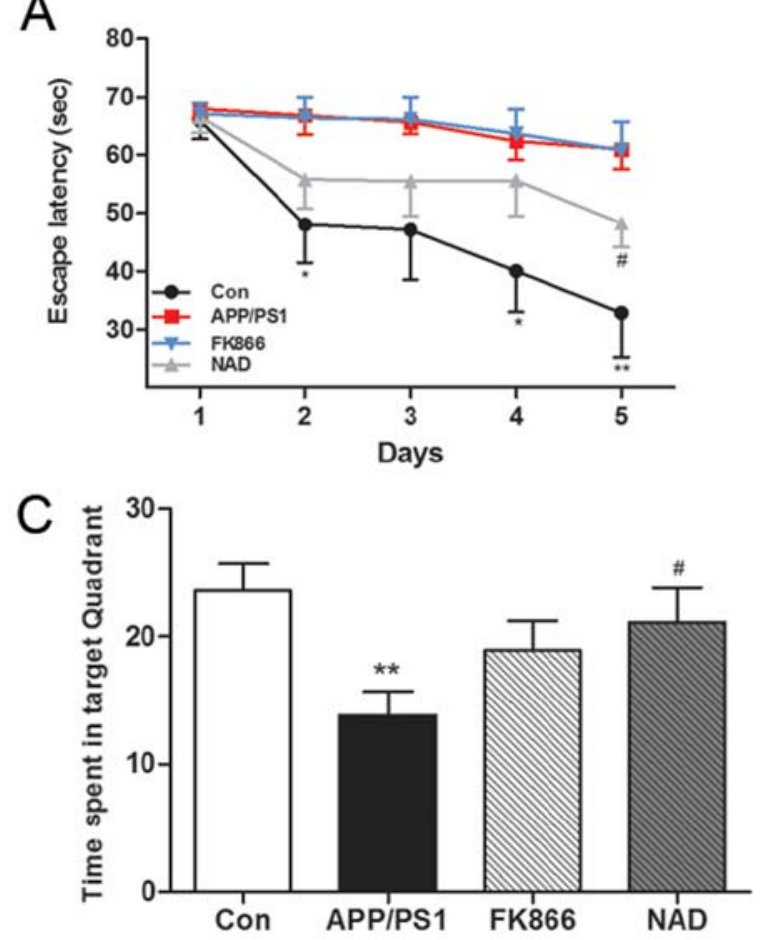

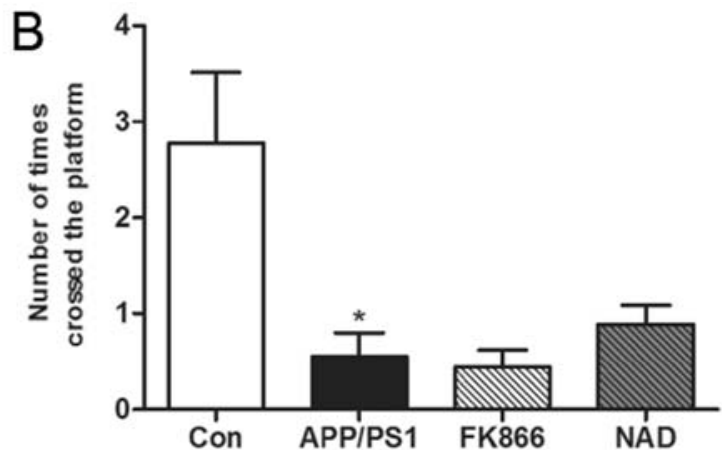

Figure 1. APP/PS1 mice exhibit decreased performance and effects of NAD ${ }^{+}$and FK866 treatment in the Morris water maze tasks. (A) Escape latency (latency to find the hidden platform) was determined in a 5-day trial study. Day 6 was a probe trial, in which (B) the number of platform crossings and (C) time spent in the target quadrant were determined (total time of the trial was $70 \mathrm{sec}$ ). Data are expressed as the mean $\pm \mathrm{standard}$ error of the mean. $\mathrm{n}=8$. ${ }^{*} \mathrm{P}<0.05,{ }^{* *} \mathrm{P}<0.01$ vs. Con; and ${ }^{\#} \mathrm{P}<0.05$ vs. APP/PS1 group. APP/PS1, APPswe/PS1 1 E9; NAD, nicotinamide adenine dinucleotide; FK866, an inhibitor of NAMPT; NAMPT, nicotinamide phosphoribosyltransferase; Con, control.

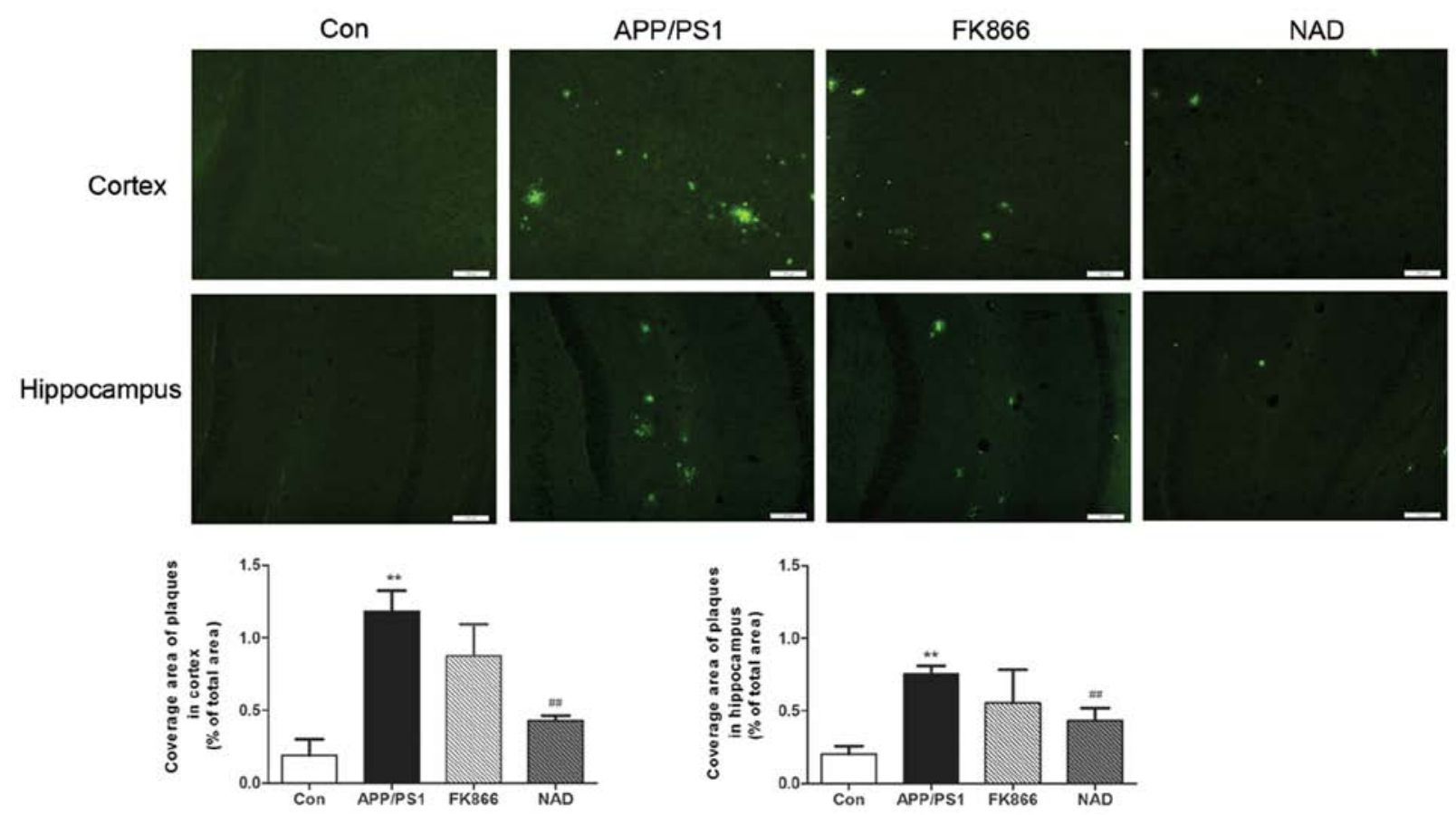

Figure 2. Histological images of Thioflavin S staining in the cortex and hippocampus and the effect of NAD ${ }^{+}$and FK866 on amyloid plaques. Scale bar, $100 \mu \mathrm{m}$. ${ }^{* *} \mathrm{P}<0.01$ vs. Con; and ${ }^{\# \#} \mathrm{P}<0.01$ vs. APP/PS1. NAD, nicotinamide adenine dinucleotide; FK866, an inhibitor of NAMPT; NAMPT, nicotinamide phosphoribosyltransferase; Con, control.

physiological conditions, the $\mathrm{NAD}^{+} / \mathrm{NADH}$ ratio reflects the redox state of cells. When the energy metabolism of the cells is deregulated, the levels of $\mathrm{NAD}^{+}$and NADH will change, which is reflected as an imbalance in the $\mathrm{NAD}^{+} / \mathrm{NADH}$ ratio (21). The present study detected $\mathrm{NAD}^{+}$and NADH levels in the cortex and hippocampus. The $\mathrm{NAD}^{+}$content in the cortex 

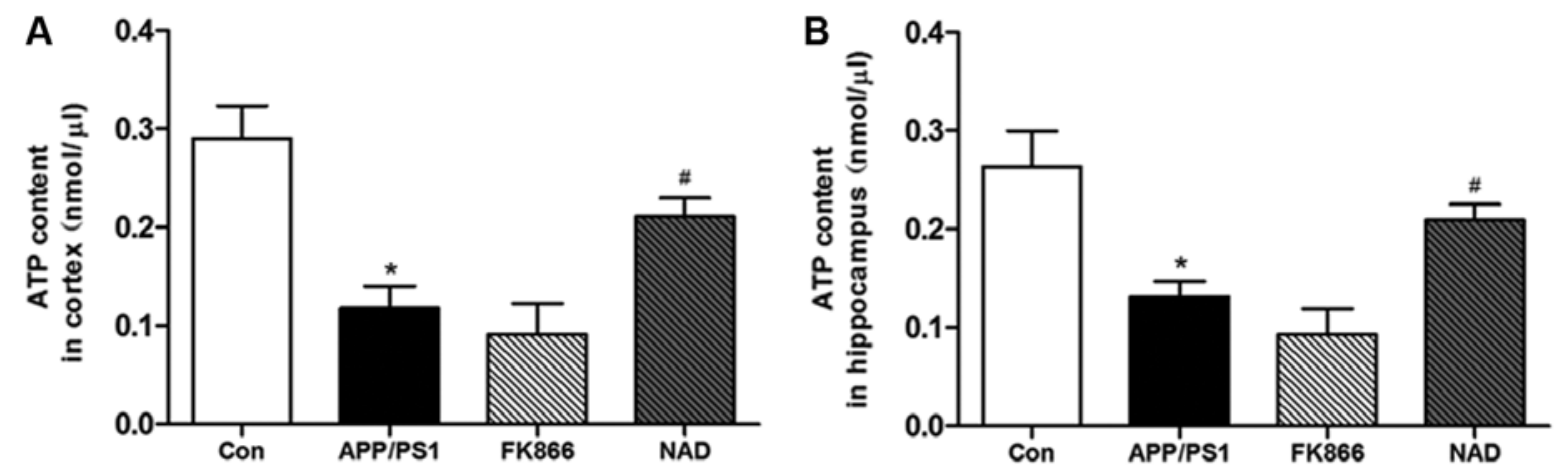

Figure 3. APP/PS1 mice exhibited decreased ATP content, and the effects of NAD ${ }^{+}$and FK866 treatment on ATP content. (A) Change of ATP content in the cortex. (B) Change of ATP content in the hippocampus. All data are expressed as the mean \pm standard error of the mean. $n=3$. " $P<0.05$, vs. Con; and ${ }^{\#} \mathrm{P}<0.05$, vs. APP/PS1. APP/PS1, APPswe/PS1 $\triangle \mathrm{E} 9$; NAD, nicotinamide adenine dinucleotide; FK866, an inhibitor of NAMPT; NAMPT, nicotinamide phosphoribosyltransferase; Con, control.

in the APP/PS1 group was significantly decreased compared with the control group $(\mathrm{P}<0.05)$ and the $\mathrm{NAD}^{+} / \mathrm{NADH}$ ratio was similarly decreased $(\mathrm{P}<0.01)$, although the NADH level was not significantly changed. Compared with the APP/PS1 model group, the $\mathrm{NAD}^{+}$content of the cortex was significantly increased in the $\mathrm{NAD}^{+}$group $(\mathrm{P}<0.05)$ and the $\mathrm{NAD}^{+} / \mathrm{NADH}$ ratio was significantly increased $(\mathrm{P}<0.01)$, while the NADH level was not significantly changed. Separately, in comparison with APP/PS1 mice, $\mathrm{NAD}^{+}$content in the cortex decreased significantly in the FK866 group $(\mathrm{P}<0.05)$ and the $\mathrm{NAD}^{+} / \mathrm{NADH}$ ratio decreased $(\mathrm{P}<0.01)$, although the NADH level did not change significantly between the two groups.

Compared with the control group, the $\mathrm{NAD}^{+}$content in the hippocampus in the APP/PS1 group was significantly decreased $(\mathrm{P}<0.05)$, while the NADH level was not significantly changed and the $\mathrm{NAD}^{+} / \mathrm{NADH}$ ratio was decreased $(\mathrm{P}<0.01)$. Furthermore, compared with the APP/PS1 model group, the $\mathrm{NAD}^{+}$content in the hippocampus in the $\mathrm{NAD}^{+}$group was significantly increased $(\mathrm{P}<0.05)$ and the $\mathrm{NAD}^{+} / \mathrm{NADH}$ ratio was significantly increased $(\mathrm{P}<0.01)$, while the NADH level was not significantly different. Regarding the $\mathrm{NAD}^{+}$content, $\mathrm{NADH}$ level and $\mathrm{NAD}^{+} / \mathrm{NADH}$ ratio, there was no significant change in the hippocampus in the FK866 group compared with the other mice (Fig. 4).

Expression of NAMPT protein decreases in APP/PS1 transgenic mice and $N A D^{+}$treatment improves the expression. In order to confirm the expression of NAMPT proteins in the cortex and hippocampus in APP/PS1 transgenic mice, immunohistochemical staining was performed. It was observed that the brown positive expression of NAMPT protein in the cortex and hippocampus in APP/PS1 group was decreased compared with the control group $(\mathrm{P}<0.05)$. Following intraperitoneal injection of $\mathrm{NAD}^{+}$, the positive expression of NAMPT protein increased compared with the APP/PS1 group $(\mathrm{P}<0.05)$, while there was no significant change in NAMPT levels in the cortex and hippocampus in FK866 mice compared with APP/PS1 mice (P>0.05; Fig. 5).

Protein extracts were also prepared and analyzed by western blotting to determine protein levels. The results demonstrated that the protein expression of NAMPT and SIRT1 in the cortex in the APP/PS1 group was significantly decreased when compared with the control group $(\mathrm{P}<0.01)$. Intraperitoneally injecting $\mathrm{NAD}^{+}$increased the expression of NAMPT in the cortex in the $\mathrm{NAD}^{+}$mice $(\mathrm{P}<0.05)$. There was no significant change in NAMPT protein levels in the FK866 mice compared with in APP/PS1 mice $(\mathrm{P}>0.05)$. Consistent with the changes in the cortex, the results demonstrated that the NAMPT and SIRT1 level in the hippocampus in the APP/PS1 group was decreased compared with the control group $(\mathrm{P}<0.01)$. NAMPT expression levels in the hippocampus in the $\mathrm{NAD}^{+}$group were also significantly increased after intraperitoneal injection of $\mathrm{NAD}^{+}(\mathrm{P}<0.05)$; however, injecting $\mathrm{NAD}^{+}$did not increase SIRT1 expression levels. Finally, there was no significant change in NAMPT and SIRT1 protein expression levels in the hippocampus in FK866 mice, compared with in APP/PS1 mice ( $\mathrm{P}>0.05$; Fig. 6$)$.

\section{Discussion}

AD is a degenerative disease of the nervous system. Studies have demonstrated that energy metabolism abnormalities are closely related to the development of early AD (22-25). The energy metabolism of failing neurons often precedes, or occurs simultaneously with, cognitive impairment (26). The mechanism may be that the energy metabolism disorder of the brain can upregulate amyloid precursor protein amyloidal digestion pathway to promote the production of $A \beta$ and $A \beta$ deposition, further causing mitochondrial dysfunction and leading to abnormal energy metabolism $(27,28)$. In addition, abnormal energy metabolism of the brain has also been associated with hyperphosphorylation of the protein $\tau$, which can affect synaptic function and aggravate cognitive impairment by activating the p38/mitogen-activated protein kinase-related pathway (29).

$\mathrm{NAD}^{+}$is required for mitochondrial respiration and ATP synthesis and maintenance of $\mathrm{NAD}^{+}$level is important for the prevention of mitochondrial dysfunction (30). $\mathrm{NAD}^{+}$level declines with age in many cell and tissue types, with altered $\mathrm{NAD}^{+}$metabolism and concurrent alterations in the mitochondrial function being inherent in $\mathrm{AD}(31)$. NAD ${ }^{+}$can be synthesized via different precursors; the main two synthetic pathways are tryptophan synthetic pathways de novo and salvage pathways $(32,33)$. The de novo synthesis pathway of 
A

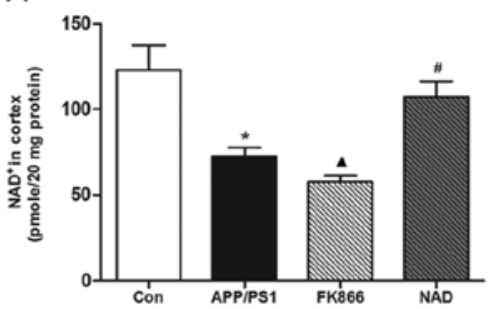

D

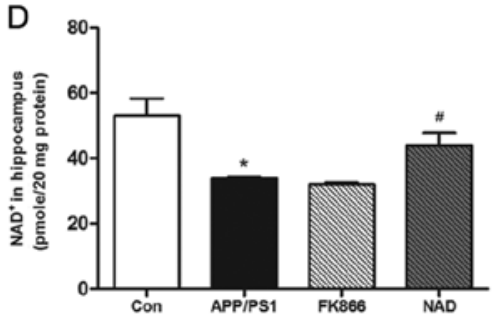

B

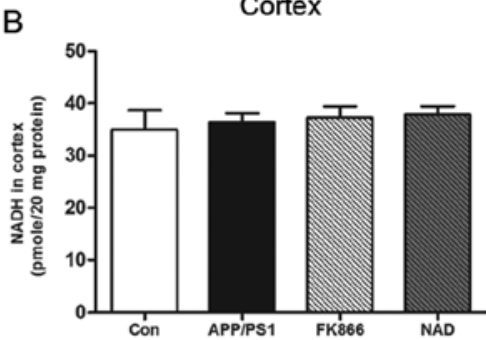

Hippocampus

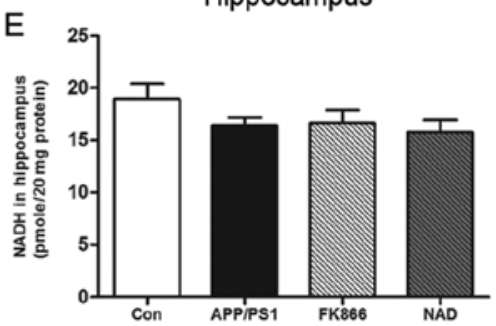

C

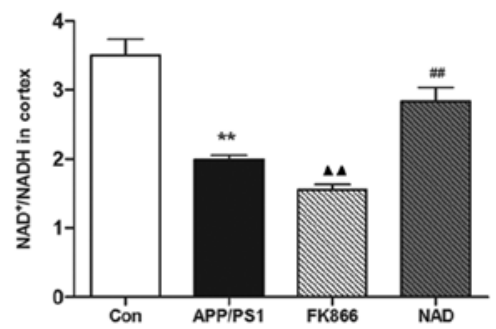

$\mathrm{F}$

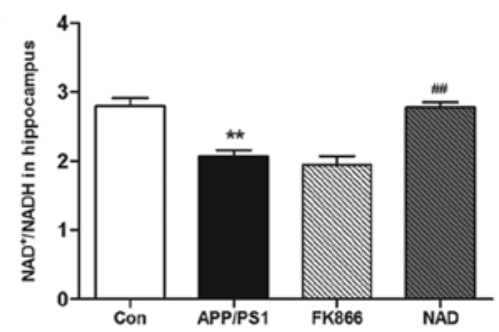

Figure 4. $\mathrm{NAD}^{+}$level and $\mathrm{NAD}^{+} / \mathrm{NADH}$ ratio of $\mathrm{APP} / \mathrm{PS} 1$ mice and effects of $\mathrm{NAD}^{+}$and FK866 treatment. (A) NAD ${ }^{+}$level, (B) NADH level and $(\mathrm{C}) \mathrm{NAD} / \mathrm{NADH}$ ratio in the cortex in every group. (D) NAD level, (E) NADH level and (F) NAD $/ \mathrm{NADH}^{+}$ratio in the hippocampus in every group. All data are expressed as the mean \pm standard error of the mean $(\mathrm{n}=4) .{ }^{*} \mathrm{P}<0.05,{ }^{* *} \mathrm{P}<0.01$ vs. Con; ${ }^{*} \mathrm{P}<0.05,{ }^{\# \#} \mathrm{P}<0.01$ vs. APP/PS1; and ${ }^{\boldsymbol{\Delta}} \mathrm{P}<0.05,{ }^{\boldsymbol{\Delta}} \mathrm{P}<0.01$ vs. APP/PS1. NAD, nicotinamide adenine dinucleotide; APP/PS1, APPswe/PS1 $\triangle \mathrm{E} 9$; Con, control.
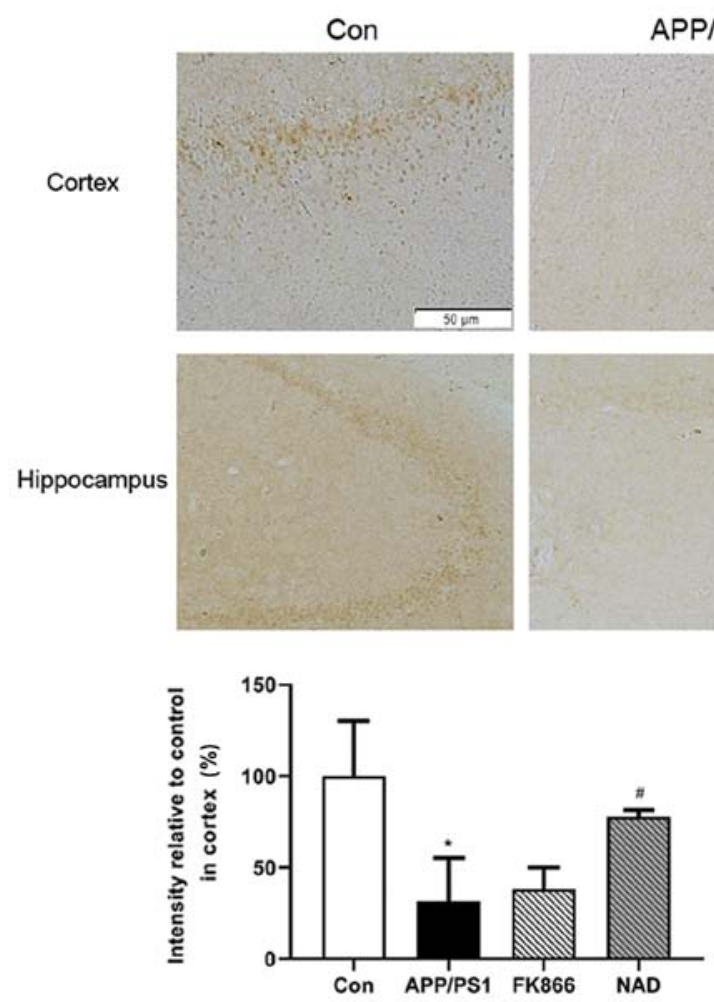

FK866
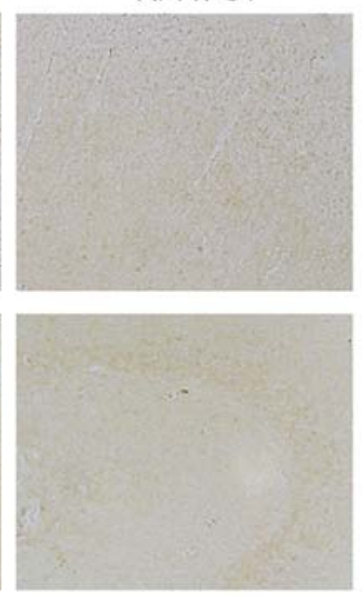
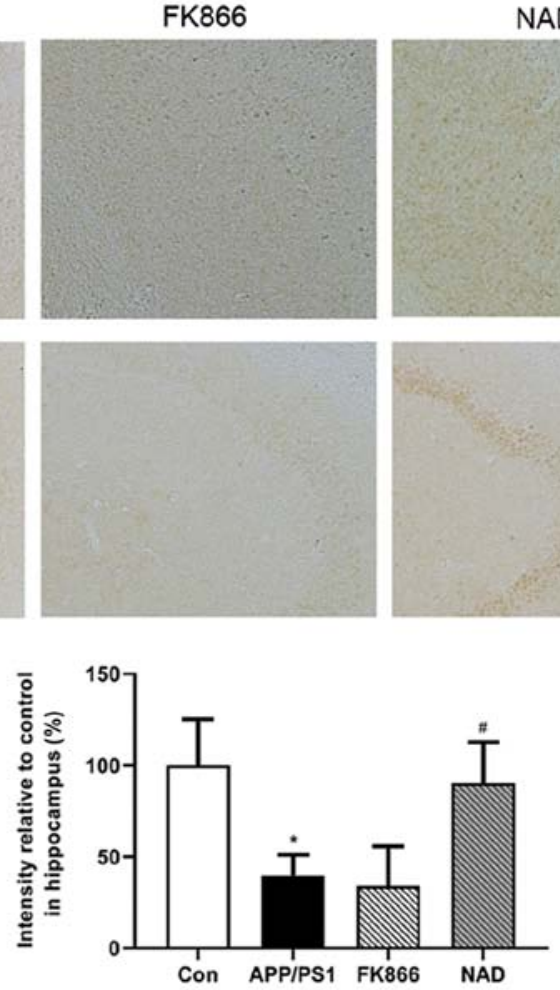

Figure 5. Immunohistochemical staining of NAMPT expression in the cortex and hippocampus with representative images. Scale bar, $50 \mu \mathrm{m}$. "P<0.05 vs. Con; and ${ }^{\#} \mathrm{P}<0.05$ vs. APP/PS1. NAMPT, nicotinamide phosphoribosyltransferase; Con, control; APP/PS1, APPswe/PS1 $\triangle E 9$; FK866, an inhibitor of NAMPT; NAD, nicotinamide adenine dinucleotide.

tryptophan is mainly carried out in the liver and kidneys, which only synthesizes a very small amount of $\mathrm{NAD}^{+}$. The salvage pathway, using nicotinic acid (NA) and nicotinamide (NAM) as raw materials, is the main source of $\mathrm{NAD}^{+}$synthesis. NA and NAM produce nicotinamide mononucleotide (NMN) through catalyzation by nicotinic acid phosphoribosyltransferase. NAMPT, originally identified as pre-B-cell colony enhancing factor, is the rate-limiting enzyme that catalyzes the first step in the biosynthesis of $\mathrm{NAD}^{+}$from nicotinamide $(33,34)$. Inhibition of NAMPT results in significant depletion of $\mathrm{NAD}^{+}$which can modulate the activity of the TCA cycle and most of sirtuins (35).

Previous studies have found that NAD ${ }^{+}$precursors such as NMN and NAM can improve the learning and memory abilities and mitochondrial function of AD model mice, suggesting 

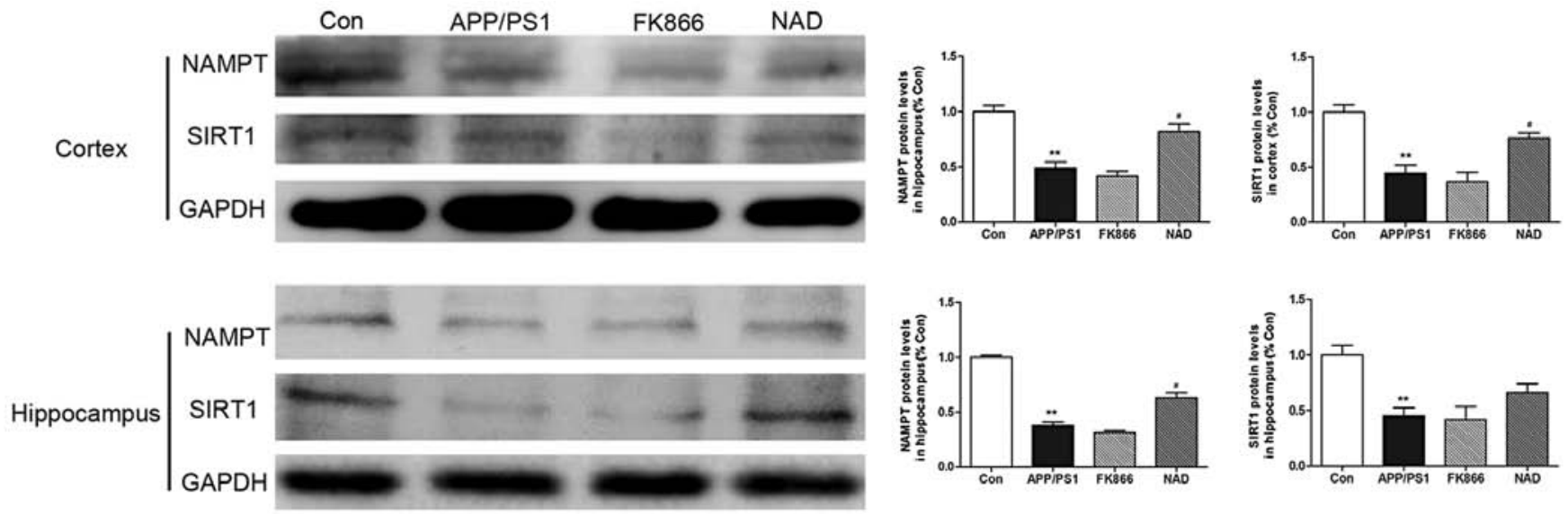

Figure 6. Western blot analysis of NAMPT and SIRT1 protein expression in the cortex and hippocampus in every group and the quantification of NAMPT protein. Data are presented as the mean \pm standard error of the mean $(n=3) .{ }^{* *} \mathrm{P}<0.01$ vs. Con; and ${ }^{~} \mathrm{P}<0.05$ vs. APP/PS1. NAMPT, nicotinamide phosphoribosyltransferase; SIRT, sirtuin; Con, control; APP/PS1, APPswe/PS1 $\triangle$ E9; FK866, an inhibitor of NAMPT; NAD, nicotinamide adenine dinucleotide.

that $\mathrm{NAD}^{+}$upregulation can reverse the damage of brain energy metabolism $(36,37)$. Other studies have suggested that $\mathrm{NAD}^{+}$mediates a number of major biological processes, including calcium homeostasis, mitochondrial functions and energy metabolism, aging, and cell death in various tissues including the brain (38-40). $\mathrm{NAD}^{+}$acts as a neuroprotective agent via several mechanisms, including the prevention of ATP depletion (41) NAMPT, a cytokine secreted by fat cells, is expressed in various tissues including the liver, kidneys, skeletal muscle and brain tissue and is involved in the oxidation of cells due to the biosynthesis of nicotinamide adenine dinucleotide (42). NAMPT affects energy metabolism, protein modification, DNA repair and other important processes by participating in the synthesis of $\mathrm{NAD}^{+}$. As a rate-limiting enzyme of the NAD ${ }^{+}$salvage pathway, NAMPT can affect cell metabolism and $\mathrm{NAD}^{+}$synthesis, interfering with $\mathrm{NAD}^{+}$ dependent-related protein expression $(38,42)$. Recently, studies have demonstrated that NAMPT is associated with central nervous system diseases, in that it can protect neurons and delay the occurrence of axonal degeneration $(43,44)$. With increasing age, the expression of NAMPT in the brain of $\mathrm{AD}$ patients is decreased, causing the level of $\mathrm{NAD}^{+}$and the antioxidant enzyme glutathione to decrease, aggravating neuronal degeneration $(45,46)$. Previous studies have demonstrated that NAMPT is mainly expressed in neurons in the mouse brain (47-49). With aging, the essential cofactor $\mathrm{NAD}^{+}$ decreases; the age-associated decrease in $\mathrm{NAD}^{+}$levels is due to a decline in protein level NAMPT (50) and AD is an age-related neurodegenerative disease (51).

The present study has demonstrated that cortex and hippocampal NAD levels and NAMPT expression declined in early stage AD model mice. Based on previous studies $(52,53)$, it is hypothesized that $\mathrm{NAD}^{+}$administration could combat NAMPT decrease in the early stage of AD. The present study demonstrated that the decrease of NAMPT lead to NAD ${ }^{+}$decrease, while NADH levels were not affected, leading to a decrease in the NAD/NADH ratio. Maintenance of $\mathrm{NAD}^{+} / \mathrm{NADH}$ ratio is critical for mitochondrial function, such as ATP synthesis in TCA cycle and oxidative phosphorylation in mitochondria (54), and it was identified that ATP decreased in the cortex and hippocampus. As $\mathrm{NAD}^{+}$is able to travel freely inside the cell, decreased cellular $\mathrm{NAD}^{+}$levels may result in reduced activity of the enzymes that use $\mathrm{NAD}^{+}$as a substrate, including SIRT1 (55). In various animal models, age-related metabolic decline is positively correlated with the decline of $\mathrm{NAD}^{+}$, and the change of $\mathrm{NAD}^{+} / \mathrm{NADH}$ ratio can regulate sirtuin enzymes, including SIRT1. SIRT1 is expressed in both brain and peripheral tissues, and may shuttle to the cytoplasm during neuronal differentiation and neurite outgrowth and apoptosis. SIRT1 has an essential role in regulating cellular homeostasis by influencing neuron survival and mitochondrial biogenesis $(56,57)$. The present study demonstrated that the intraperitoneal injection $\mathrm{NAD}^{+}$ levels in APP/PS1 mice was also associated with increased level of SIRT1. The NAD ${ }^{+}$level in APP/PS1 mice after treatment with FK866 was measured and, as expected, FK866 treated APP/PS1 mice contained significantly less total $\mathrm{NAD}^{+}$in the cortex and a lower $\mathrm{NAD}^{+} / \mathrm{NADH}$ ratio compared with APP/PS1 mice without any treatment. In the hippocampus, there was no significance difference between the FK866 and APP/PS1 groups in the level of $\mathrm{NAD}^{+}$and the $\mathrm{NAD}^{+} / \mathrm{NADH}$ ratio, which may be related to the pathological development of early $\mathrm{AD}$ mice model. However, it is considered that these results indicated that NAMPT inhibition caused $\mathrm{NAD}^{+}$deficiency, which demonstrated that improvement of NAMPT can increase the generation NAD.

The present study demonstrated that $\mathrm{NAD}^{+}$depletion with decreased NAMPT is reversible and that $\mathrm{NAD}^{+}$replenishment can reverse the impaired brain-energy metabolism that are implicated in cognitive decline and mitigates neurodegeneration. Given that NAMPT plays an important role in NAD synthesis, combining $\mathrm{NAD}^{+}$or a drug increasing NAMPT may constitute a therapy for $\mathrm{AD}$ in the future.

\section{Acknowledgements}

Not applicable.

\section{Funding}

Funding was provided by the National Natural Science Foundation of China (grant no. 81503626) and the Shanghai Health Bureau Youth Fund (grant no. 201540254). 


\section{Availability of data and materials}

The datasets used and/ or analyzed during the present study are available from the corresponding author on reasonable request.

\section{Authors' contributions}

SX was the principal investigator and designed the study. YH performed statistical analysis, conducted the study and wrote the manuscript. XH performed experiments. DS performed statistical analysis and data collection. CC contributed to experimental design and manuscript preparation. All authors have read and approved the final manuscript.

\section{Ethics approval and consent to participate}

All procedures were performed in accordance with the Guide for and Use of Medical Laboratory Animals (Ministry of Health of China, 1998) and approved by the Shanghai University of Traditional Chinese Medicine Laboratory Animal Care and Use Committee (no. PZSHUTCM19011807).

\section{Patient consent for publication}

Not applicable.

\section{Competing interests}

The authors declare that they have no competing interests.

\section{References}

1. Hardy J and Selkoe DJ: The amyloid hypothesis of Alzheimer's disease: Progress and problems on the road to therapeutics. Science 297: 353-356, 2002.

2. Yin F, Boveris A and Cadenas E: Mitochondrial energy metabolism and redox signaling in brain aging and neurodegeneration. Antioxid Redox Signal 20: 353-371, 2014.

3. Yin F, Sancheti H, Patil I and Cadenas E: Energy metabolism and inflammation in brain aging and Alzheimer's disease. Free Radic Biol Med 100: 108-122, 2016.

4. Cabezas-Opazo FA, Vergara-Pulgar K, Pérez MJ, Jara C, Osorio-Fuentealba C and Quintanilla RA: Mitochondrial dysfunction contributes to the pathogenesis of Alzheimer's disease. Oxid Med Cell Longev 2015: 509654, 2015.

5. Han XJ, Hu YY, Yang ZJ, Jiang LP, Shi SL, Li YR, Guo MY, Wu HL and Wan YY: Amyloid $\beta$-42 induces neuronal apoptosis by targeting mitochondria. Mol Med Rep 16: 4521-4528, 2017.

6. Wang R, Li JJ, Diao S, Kwak YD, Liu L, Zhi L, Büeler H, Bhat NR, Williams RW, Park EA and Liao FF: Metabolic stress modulates Alzheimer's $\beta$-secretase gene transcription via SIRT1-PPARY-PGC-1 in neurons. Cell Metab 17: 685-694, 2013.

7. Wu MF, Yin JH, Hwang CS, Tang CM and Yang DI: NAD attenuates oxidative DNA damages induced by amyloid beta-peptide in primary rat cortical neurons. Free Radic Res 48: 794-805, 2014.

8. Seixas da Silva GS, Melo HM, Lourenco MV, Lyra E Silva NM, de Carvalho MB, Alves-Leon SV, de Souza JM, Klein WL, da-Silva WS, Ferreira ST and De Felice FG: Amyloid- $\beta$ oligomers transiently inhibit AMP-activated kinase and cause metabolic defects in hippocampal neurons. J Biol Chem 292: 7395-7406, 2017.

9. Rijpma A, van der Graaf M, Meulenbroek O, Olde Rikkert MGM and Heerschap A: Altered brain high-energy phosphate metabolism in mild Alzheimer's disease: A 3-dimensional ${ }^{31} \mathrm{P}$ MR spectroscopic imaging study. Neuroimage Clin 18: 254-261, 2018.

10. Rajmohan R and Reddy PH: Amyloid-beta and phosphorylated tau accumulations cause abnormalities at synapses of Alzheimer's disease Neurons. J Alzheimers Dis 57: 975-999, 2017.
11. Elhassan YS, Philp AA and Lavery GG: Targeting NAD+ in metabolic disease: New insights into an old molecule. J Endocr Soc 1: 816-835, 2017.

12. Rongvaux A, Shea RJ, Mulks MH, Gigot D, Urbain J, Leo O and Andris F: Pre-B-cell colony-enhancing factor, whose expression is up-regulated in activated lymphocytes, is a nicotinamide phosphoribosyltransferase, a cytosolic enzyme involved in NAD biosynthesis. Eur J Immunol 32: 3225-3234, 2002.

13. Garten A, Schuster S, Penke M, Gorski T, de Giorgis T and Kiess W: Physiological and pathophysiological roles of NAMPT and NAD metabolism. Nat Rev Endocrinol 11: 535-546, 2015.

14. Ruggieri S, Orsomando G, Sorci L and Raffaelli N: Regulation of NAD biosynthetic enzymes modulates NAD-sensing processes to shape mammalian cell physiology under varying biological cues. Biochim Biophys Acta 1854: 1138-1149, 2015.

15. Verdin $\mathrm{E}: \mathrm{NAD}^{+}$in aging, metabolism, and neurodegeneration. Science 350: 1208-1213, 2015.

16. Zhang Y,Zhang Y, Li X, Zhang M and Lv K: Microarray analysis of circular RNA expression patterns in polarized macrophages. Int J Mol Med 39: 373-379, 2017.

17. Trinchese F, Liu S, Battaglia F, Walter S, Mathews PM and Arancio O: Progressive age-related development of alzheimer-like pathology in app/ps1 mice. Ann Neurol 55: 801-814, 2004.

18. Janus C, Flores AY, Xu G and Borchelt DR: Behavioral abnormalities in app swe /pslde9 mouse model of ad-like pathology: Comparative analysis across multiple behavioral domains. Neurobiol Aging 36: 2519-2532, 2015.

19. Balducci C, Mancini S, Minniti S, La Vitola P, Zotti M, Sancini G, Mauri M, Cagnotto A, Colombo L, Fiordaliso F, et al: Multifunctional liposomes reduce brain $\beta$-amyloid burden and ameliorate memory impairment in Alzheimer's disease mouse models. J Neurosci 34: 14022-14031, 2014.

20. Caccamo A, Branca C, Talboom JS, Shaw DM, Turner D, Ma L, Messina A, Huang Z, Wu J and Oddo S: Reducing ribosomal protein S6 Kinase 1 expression improves spatial memory and synaptic plasticity in a mouse model of Alzheimer's disease. J Neurosci 35: 14042-14056, 2015.

21. Kim SY, Cohen BM, Chen X, Lukas SE, Shinn AK, Yuksel AC, Li T, Du F and Öngür D: Redox Dysregulation in schizophrenia revealed by in vivo NAD+/NADHH measurement. Schizophr Bull 43: 197-204, 2017.

22. Blass JP, Sheu RK and Gibson GE: Inherent abnormalities in energy metabolism in Alzheimer disease. Interaction with cerebrovascular compromise. Ann N Y Acad Sci 903: 204-221, 2000.

23. Lewczuk P, Riederer P, O'Bryant SE, Verbeek MM, Dubois B, Visser PJ, Jellinger KA, Engelborghs S, Ramirez A, Parnetti L, et al: Cerebrospinal fluid and blood biomarkers for neurodegenerative dementias: An update of the consensus of the task force on biological markers in psychiatry of the world federation of societies of biological psychiatry. World J Biol Psychiatry 19: 244-328, 2018.

24. Pérez MJ,Ponce DP, Aranguiz A, Behrens MI and Quintanilla RA: Mitochondrial permeability transition pore contributes to mitochondrial dysfunction in fibroblasts of patients with sporadic Alzheimer's disease. Redox Biol 19: 290-300, 2018.

25. Swerdlow RH, Koppel S, Weidling I, Hayley C, Ji Y and Wilkins HM: Mitochondria, Cybrids, Aging, and Alzheimer's disease. Prog Mol Biol Transl Sci 146: 259-302, 2017.

26. Adiele RC and Adiele CA: Mitochondrial regulatory pathways in the pathogenesis of Alzheimer's disease. J Alzheimers Dis 53: 1257-1270, 2016.

27. Velliquette RA, O'ConnorT and Vassar R: Energy inhibition elevates beta-secretase levels and activity and is potentially amyloidogenic in APP transgenic mice: Possible early events in Alzheimer's disease pathogenesis. J Neurosci 25: 10874-10883, 2005.

28. Wu Z, Zhu Y, Cao X, Sun S and Zhao B: Mitochondrial toxic effects of $A \beta$ through mitofusins in the early pathogenesis of Alzheimer's disease. Mol Neurobiol 50: 986-996, 2014.

29. Lauretti E, Li JG, Di Meco A and Pratico D: Glucose deficit triggers tau pathology and synaptic dysfunction in a tauopathy mouse model. Transl Psychiatry 7: e1020, 2017.

30. Fang EF: Mitophagy and NAD ${ }^{+}$inhibit Alzheimer disease. Autophagy 15: 1112-1114, 2019.

31. Ghosh D, Levault KR and Brewer GJ: Relative importance of redox buffers GSH and NAD+(P)H in age-related neurodegeneration and Alzheimer disease-like mouse neurons. Aging Cell 13: 631-640, 2014.

32. Yang $\mathrm{Y}$ and Sauve AA: $\mathrm{NAD}(+)$ metabolism: Bioenergetics, signaling and manipulation for therapy. Biochim Biophys Acta 1864: 1787-1800, 2016. 
33. Pehar M, Harlan BA, Killoy KM and Vargas MR: Nicotinamide adenine dinucleotide metabolism and Neurodegeneration. Antioxid Redox Signal 18: 1652-1668, 2018.

34. Stein LR and Imai S: The dynamic regulation of NAD metabolism in mitochondria. Trends Endocrinol Metab 23: 420-428, 2012.

35. Long AN, Owens K, Schlappal AE, Kristian T, Fishman PS and Schuh RA: Effect of nicotinamide mononucleotide on brain mitochondrial respiratory deficits in an Alzheimer's disease-relevant murine model. BMC Neurol 15: 19, 2015.

36. Yao Z, Yang W, Gao Z and Jia P: Nicotinamide mononucleotide inhibits JNK activation to reverse Alzheimer disease. Neurosci Lett 647: 133-140, 2017.

37. Wang X, Zhang Q, Bao R, Zhang N, Wang Y, Polo-Parada L, Tarim A, Alemifar A, Han X, Wilkins HM, et al: Deletion of NAMPT in projection neurons of adult mice leads to motor dysfunction, Neurodegeneration, and death. Cell Rep 20: 2184-2200, 2017.

38. Katsyuba E and Auwerx J: Modulating $\mathrm{NAD}^{+}$metabolism, from bench to bedside. EMBO J 36: 2670-2683, 2017.

39. Nikiforov A, Kulikova V and Ziegler M: The human NAD metabolome: Functions, metabolism and compartmentalization. Crit Rev Biochem Mol Biol 50: 284-297, 2015.

40. Ryu KW, Nandu T, Kim J, Challa S, DeBerardinis RJ and Kraus WL: Metabolic regulation of transcription through compartmentalized $\mathrm{NAD}^{+}$biosynthesis. Science 360: pii: eaan5780, 2018.

41. Zhu XH, Lu M, Lee BY, Ugurbil $\mathrm{K}$ and Chen W: In vivo NAD assay reveals the intracellular NAD contents and redox state in healthy human brain and their age dependences. Proc Natl Acad Sci USA 112: 2876-2881, 2015.

42. Revollo JR, Grimm AA and Imai S: The regulation of nicotinamide adenine dinucleotide biosynthesis by Nampt/PBEF/visfatin in mammals. Curr Opin Gastroenterol 23: 164-170, 2007.

43. Lin JB, Kubota S, Ban N, Yoshida M, Santeford A, Sene A, Nakamura R, Zapata N, Kubota M, Tsubota K, et al: NAMPT-Mediated NAD(+) Biosynthesis is essential for vision in mice. Cell Rep 17: 69-85, 2016.

44. Yamamoto T, Byun J, Zhai P, Ikeda Y, Oka S and Sadoshima J: Nicotinamide mononucleotide, an intermediate of NAD+ synthesis, protects the heart from ischemia and reperfusion. PLoS One 9: e98972, 2014.

45. LoCoco PM, Risinger AL, Smith HR, Chavera TS, Berg KA and Clarke WP: Pharmacological augmentation of nicotinamide phosphoribosyltransferase (NAMPT) protects against paclitaxel-induced peripheral neuropathy. Elife 6: e29626, 2017.

46. Ghosh D, LeVault KR, Barnett AJ and Brewer GJ: A reversible early oxidized redox state that precedes macromolecular ROS damage in aging nontransgenic and 3xTg-AD mouse neurons. J Neurosci 32: 5821-5832, 2012.
47. Sasaki Y, Araki T and Milbrandt J: Stimulation of nicotinamide adenine dinucleotide biosynthetic pathways delays axonal degeneration after axotomy. J Neurosci 26: 8484-8491, 2006.

48. Chen J, Sysol JR, Singla S, Zhao S, Yamamura A, Valdez-Jasso D, Abbasi T, Shioura KM, Sahni S, Reddy V, et al: Nicotinamide Phosphoribosyltransferase promotes pulmonary vascular remodeling and is a therapeutic target in pulmonary arterial hypertension. Circulation 135: 1532-1546, 2017.

49. Wang SN and Miao CY: Targeting NAMPT as a therapeutic strategy against stroke. Stroke Vasc Neurol 4: 83-89, 2019.

50. Imai S and Yoshino J: The importance of NAMPT/NAD/SIRT1 in the systemic regulation of metabolism and aging. Diabetes Obes Metab 15 (Suppl 3): S26-S33, 2013.

51. Stein LR and Imai S: Specific ablation of Nampt in adult neural stem cells recapitulates their functional defects during aging. EMBO J 33: 1321-1340, 2014

52. Kiss G, Konrad C, Pour-Ghaz I, Mansour JJ, Németh B, Starkov AA, Adam-Vizi V and Chinopoulos C: Mitochondrial diaphorases as NAD+ donors to segments of the cirtric acid cycle that support substrate-level phosphorylation yielding ATP during respiratory inhibition. FASEB J 28: 1682-1697, 2014.

53. Hou Y, Lautrup S, Cordonnier S, Wang Y, Croteau DL, Zavala E, Zhang Y, Moritoh K, O'Connell JF, Baptiste BA, et al: NAD+ supplementation normalizes key Alzheimer's features and DNA damage responses in a new AD mouse model with introduced DNA repair deficiency. Proc Natl Acad Sci USA 115: 1876-1885, 2018.

54. Wang X, Li H and Ding S: The effects of NAD+ on apoptotic neuronal death and mitochondrial biogenesis and function after glutamate Excitotoxicity. Int J Mol 15: 20449-20468, 2014.

55. Guan Y, Wang SR, Huang XZ, Xie QH, Xu YY, Shang D and Han CM: Nicotinamide Mononucleotide, an NAD+ Precursor, Rescues Age-associated susceptibility to AKI in a sirtuin 1-dependent manner. J Am Soc Nephrol 28: 2337-2352, 2017.

56. Du LL, Xie JZ, Cheng XS, Li XH, Kong FL, Jiang X, Ma ZW, Wang JZ, Chen C and Zhou XW: Activation of sirtuin 1 attenuates cerebral ventricular streptozotocin-induced tau hyperphosphorylation and cognitive injuries in rat hippocampi. Age (Dordr) 36: 613-623, 2014.

57. Bonfili L, Cecarini V, Cuccioloni M, Angeletti M, Berardi S, Scarpona S, Rossi G and Eleuteri AM: SLAB51 probiotic formulation activates SIRT1 pathway promoting antioxidant and neuroprotective effects in an AD mouse model. Mol Neurobiol 55: 7987-8000, 2018

This work is licensed under a Creative Commons Attribution-NonCommercial-NoDerivatives 4.0 International (CC BY-NC-ND 4.0) License. 